http://jmscr.igmpublication.org/home/ ISSN (e)-2347-176x ISSN (p) 2455-0450 crossref DOI: https://dx.doi.org/10.18535/jmscr/v8i8.29

\author{
Dournal Of Medical Science And Clinical Research \\ IGM Publication \\ An Official Publication of IGM Publication
}

\title{
Challenges in the postoperative period following resection of a massive retroperitoneal sa rcoma: A case report
}

\author{
Authors
}

\author{
Anila V B ${ }^{1}$, Karthika Asokan ${ }^{2}$, Rajasree O $^{3}$, Nimmy George ${ }^{4 *}$, Shalina Chandran ${ }^{5}$ \\ ${ }^{1}$ Postgraduate student, Department of Anaesthesiology, Regional Cancer Centre, Trivandrum \\ ${ }^{2}$ Intensivist, Department of Anaesthesiology, Regional Cancer Centre, Trivandrum \\ ${ }^{3}$ Associate Professor, Department of Anaesthesiology, Regional Cancer Centre, Trivandrum \\ ${ }^{4}$ Postgraduate student, Department of Anaesthesiology, Regional Cancer Centre, Trivandrum \\ ${ }^{5}$ Senior resident, Department of Anaesthesiology, Regional Cancer Centre, Trivandrum \\ *Corresponding Author
}

\section{Nimmy George}

Postgraduate student, Department of Anaesthesiology, Regional Cancer Centre, Trivandrum, India

\begin{abstract}
Intraoperative and postoperative period after major oncologic surgeries pose several challenges to the anaesthesiologist and the intensivist. This is a report of a huge retroperitoneal sarcoma for which wide excision and colo-colic anastomosis was done. The patient required massive blood transfusion during intraoperative period and had a stormy postoperative course in the intensive care unit (ICU) due to urosepsis, fecal peritonitis and fungal sepsis. Each of these complications was tackled promptly and appropriately. Fungal sepsis deserves a special mention in critically ill cancer patients due to their prolonged hospital stay and immunocompromised status.

Keywords: Retroperitoneal sarcoma; massive blood transfusion; fecal peritonitis, fungal sepsis; caspofung in; case report.
\end{abstract}

\section{Introduction}

Oncosurgeries are usually lengthy procedures involving resection of multiple structures with major blood loss and fluid shifts. They are associated with high incidence of postoperative complications and morbidities. We are reporting a case of huge retroperitoneal sarcoma for which wide excision and left colectomy with colo-colic anastomosis was done. We faced numerous challenges in the intraoperative and postoperative course. Timely and appropriate therapy goes a long way in good recovery of cancer patients.

\section{Case Report}

A 42 years old gentleman, diagnosed with retroperitoneal sarcoma, with history of hypertension was planned for wide excision of the tumour. Computed tomography of abdomen showed a large left retroperitoneal tumour of size $30 \times 20 \mathrm{~cm}$ extending from L1 to $\mathrm{S} 1$ level, infiltrating the left ureter causing left hydroureteronephrosis (Figures1,2). Left ureteral stent insertion was done preoperatively. Intraoperatively, he had a blood loss of 4 litres and massive transfusion was initiated. He received 5packed RBCs (PRBC), 4 fresh frozen plasma (FFP) and 4 platelet concentrates. He also received $20 \%$ albumin $200 \mathrm{ml}$ and 6 litres of crystalloids. Noradrenaline support was initiated to maintain normal hemodynamics. In view of dense adhesions to the mesocolon, left colectomy and colo-colic anastomosis was done. 
Postoperatively, he was electively ventilated and sedated with fentanyl infusion20 microgram per hour. He was given $200 \mathrm{ml} 20 \%$ albumin to correct his volume deficit along with crystalloids. Noradrenaline support could be tapered overnight and he was extubated the next day. On postoperative day (POD 2) he had an episode of malaena, abdominal distension, high fever spikes and tachypnoea. His total count was 3,900 but platelet count and haemoglobin were normal, serum creatinine levels rose to $1.6 \mathrm{mg} / \mathrm{dl}$ and internationalised normal ratio (INR) increased to 1.7. His procalcitonin level was high $(16 \mathrm{ng} / \mathrm{ml})$ and a diagnosis of bacterial sepsis was made. As urine culture showed growth of multi drugresistant (MDR) Klebsiella species (sensitive to colistin), Left ureteral stent which slipped into the urinary bladder during resection, was removed immediately and colistin was started. Noninvasive ventilatory support was initiated on the same day due to respiratory distress but he was intubated due to worsening acid base status. Lung ultrasound showed bilateral basal consolidation and left pleural effusion. By POD5, his total WBC picked up to 4,700 and serum creatinine and INR dropped to 1.4 and 1.2 respectively (Table 1). However the same day evening, he had recurrent fever spikes $\left(102-104^{0} \mathrm{~F}\right)$ and intravenous fosfomycin $4 \mathrm{~g}$ thrice daily was started empirically. On POD 6, abdominal ultrasound revealed ascites. A diagnostic tap was done which showed fecal peritonitis. Emergency re-exploration and reinforcement of colo-colic anastomosis with diversion ileostomy was done. Total parenteral nutrition was started and he was extubatedon POD12to NIV. He had recurrent fever spikes from POD 20 and blood culture revealed significant growth of Candida albicans from blood samples taken from central venous catheter (CVC) and peripheral vein. CVC was removed immediately and we started caspofungin. A loading dose of intravenous caspofungin $70 \mathrm{mg}$ was given on day 1 followed by $50 \mathrm{mg}$ intravenously once daily from next day. His fever spikes decreased after 2 days and he was started on oral feeds and shifted out of ICU to High dependency Unit. The repeat blood cultures after one week was sterile but intravenous caspofungin was continued for 15 days. He was discharged on POD 32 in a stable condition.

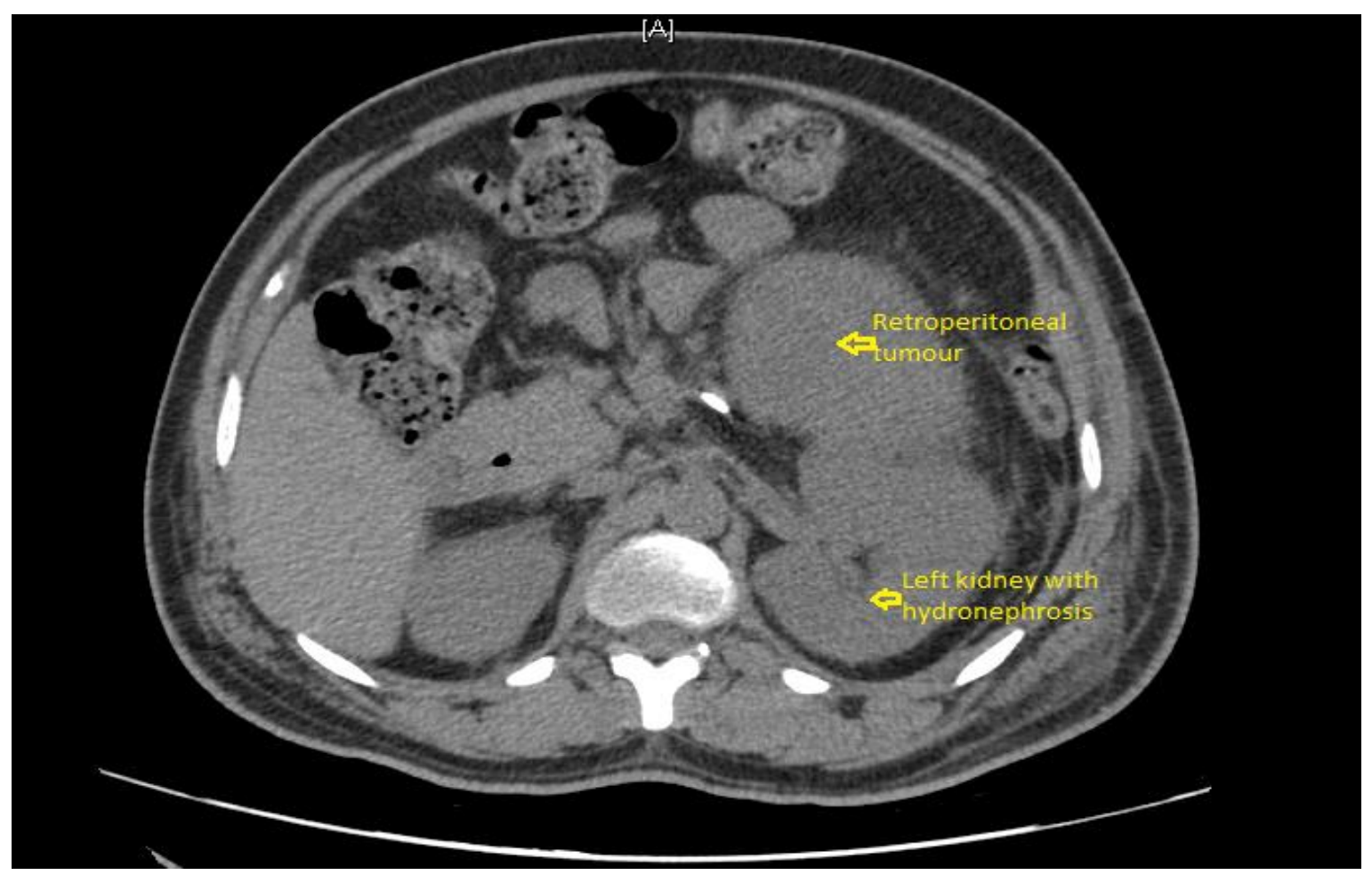

Figure 1 Computed tomography abdomen (L1 level) showing left hydronephrosis and retroperitoneal tumour. 


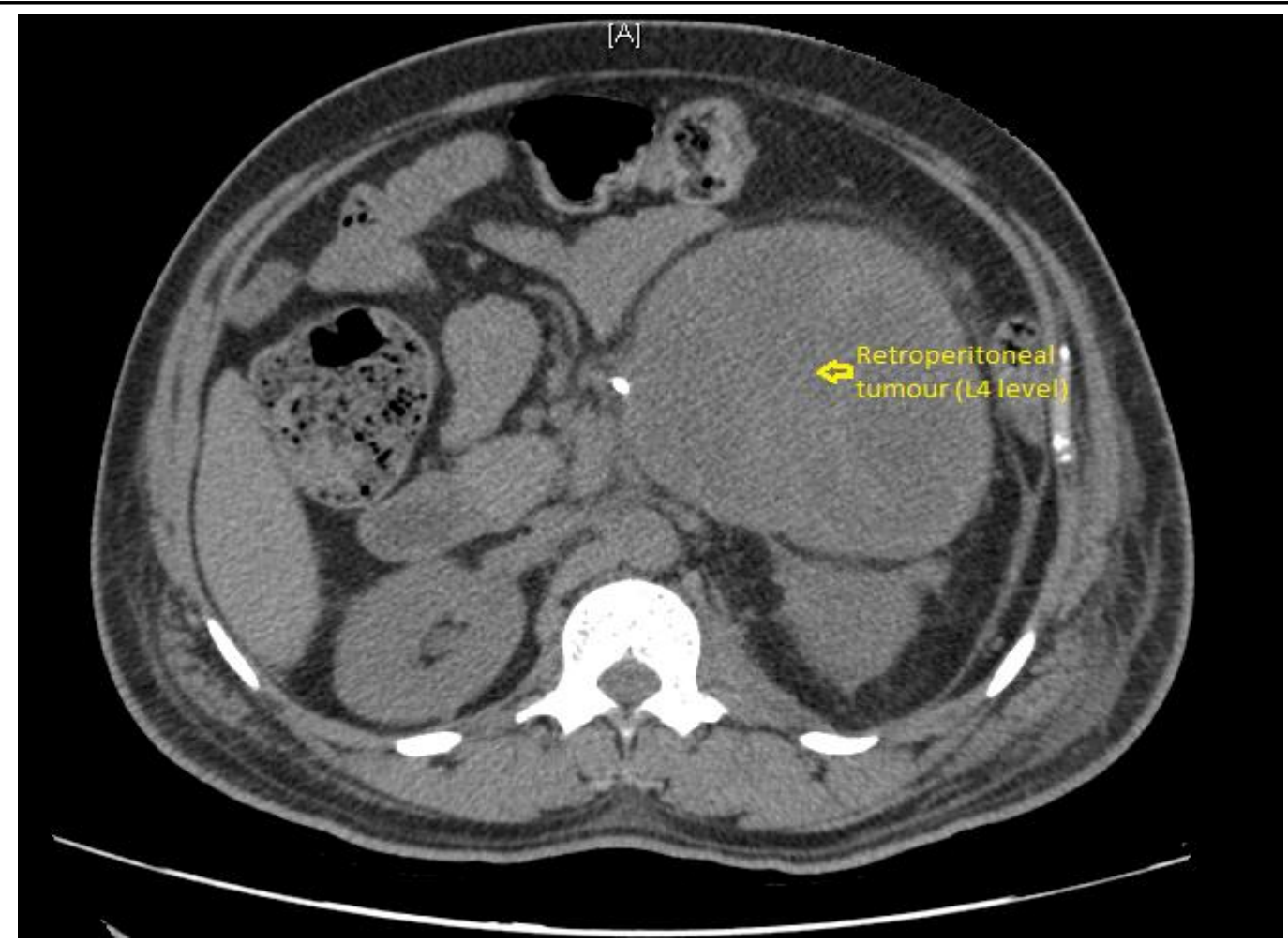

Figure 2: Computed Tomography abdomen (L3 level) showing the huge retroperitoneal tumour.

Table 1: Laboratory investigations during the postoperative period

\begin{tabular}{|l|c|c|c|c|c|c|}
\hline & POD1 & POD2 & POD3 & POD4 & POD5 & POD6 \\
\hline Hemoglobin & 9.7 & 10.6 & 11.8 & 11.1 & 11.2 & 11 \\
\hline WBC & 7900 & 3900 & 4100 & 4300 & 4700 & 5300 \\
\hline INR & 1.63 & 1.7 & 1.5 & 1.2 & 1.2 & 1.1 \\
\hline S.Creatinine & 1.2 & 1.6 & 1.7 & 1.5 & 1.4 & 1.2 \\
\hline Procalcitonin & & 16 & & & 6 & \\
\hline
\end{tabular}

\section{Discussion}

Massive blood transfusion ${ }^{(1)}$ is encountered during major oncosurgical procedures. Latest guidelines suggest the ratio of 1:1:1 for PRBC:FFP:Platelet. Immediate complications secondary to volume resuscitation, overzealous resuscitation and dilutional problems as well as delayed complications like respiratory failure, sepsis and thrombotic complications may be anticipated in the postoperative period ${ }^{(2)}$.

Ureteral stents are inserted preoperatively in abdominal tumors causing ureteric compression. Our patient had significant MDR Klebsiella growth in urine due to this intervention. Indwelling ureteral stents is associated with increased chance of urinary tract infection secondary to the formation of microbial biofilm on the stent surface. Current strategies to overcome this include frequent stent replacement, early removal and culture- specific antibiotics ${ }^{(3)}$. Preoperative urine culture and prophylactic stent removal before surgery could have avoided this complication in the postoperative period. Although our patient had a transient drop in total leucocyte count, it is not highly suggestive of sepsis. However, a substantially abnormal WBC may suggest the presence of underlying infection $^{(4)}$. Elevated procalcitonin levels, though not specific, is one of the critical tools in diagnosis of sepsis ${ }^{(5)}$.

Prolonged intensive care unit stay predisposes patients to nosocomial infections. Fungal sepsis is associated with considerable morbidity and mortality. Patients with invasive candidiasis receiving inappropriate initial therapy showed higher mortality than infections caused by bacterial pathogens in ICU settings ${ }^{(6)}$. Blot et al. ${ }^{(7)}$ observed a mortality of $78 \%$ in invasive 
candidiasis (IC) when therapy was delayed more than 48 hours from onset of candidemia, and a lower mortality of $44 \%$ in those who received adequate and prompt initial treatment. In one of the largest studies done by Ostrosky-Zeichner et al. ${ }^{(8)}$, they identified specific risk factors for IC. Major risk factors included receipt of systemic antibiotics and presence of a CVC. Minor risk factors included total parenteral nutrition, dialysis, surgery in preceding week, pancreatitis and use of steroids or immunosuppressants. If patient has major risk factors with atleast 2 of the minor risk factors, they constituted the high- risk group for IC. The calculated sensitivity was only $34 \%$ but the specificity was as high as $90 \%$. Caspofungin is an antifungal drug that belongs to the echinocandin class of drugs. The mechanism of action is via inhibition of $\beta-1,3$ - glucan synthase, an enzyme responsible for production of major cell wall component $\beta$-1,3-D-glucan. They are the most recently developed antifungals which are FDA approved for candidemia ${ }^{(9)}$. The Infectious Diseases Society of America (IDS A) recommends echinocandin for moderate to severe IC and transition from an echinocandin to fluconazole when an isolate that is likely to be susceptible to fluconazole (eg, C albicans, C tropicalis, Cparapsilosis) is identified and patients have clinically stabilized ${ }^{10}$. The IDS A also recommend removal of any intravascular catheter that is positive for a fungal pathogen based on evidence from large retrospective and observational studies. We removed the CVC that yielded candida growth in culture and started intravenous caspofungin which was continued for 15 days. The current guidelines $^{(10)}$ also suggest ophthalmologic examination for every patient with candidemia as endophthalmitis is a common complication in IC. Hence, source control that includes removal of infected lines and evaluation for endophthalmitis is essential for all patients with IC.

This case highlights the following learning points: 1) to be more watchful of chance of urosepsis in patients with ureteral stents, and 2)prompt and aggressive treatment of fungal sepsis by continuing antifungal drug for 2 weeks aids in reducing morbidity.

\section{Conclusion}

Massive tumors and their resections are indeed a challenge for the anaesthesiologist as well as the intensivist. Timely interventions can prevent significant morbidity and mortality in such cases. Fungal sepsis needs to be aggressively treated as it is associated with higher morbidity and mortality than bacterial septicaemia. Critical strategies include identifying high- risk patients, prompt antifungal therapy, choosing appropriate antifungal agent and source control. The possibility of urosepsis in patients with ureter stents should also not be overruled.

\section{Sources of support: Nil \\ Conflicts of Interest: $\mathrm{Nil}$ \\ Funding: Nil}

Acknowledgements: Patient and his relatives for consent.

\section{References}

1. Nunez TC, Young PP, Holcomb JB, Cotton BA: Creation, implementation, and maturation of a massive transfusion protocol for the exsanguinating trauma patient. J Trauma. 2010;68:1498-505.doi: 10.1097/TA.0b013e3181d3cc25

2. Patil V, Shetmahajan $\mathrm{M}$ : massive transfusion and massive transfusion protocol, Indian J Anaesth. 2014[cited 2020 Aug 11];58:590-5. doi: 10.4103/0019-5049.144662

3. Scotland KB, Lo J, Grgic T, Lange D. Ureteral stent-associated infection and sepsis: pathogenesis and prevention: a review. Biofouling. 2019;35(1):117-127. doi:10.1080/08927014.2018.1562549

4. Farkas JD. The complete blood count to diagnose septic shock. J Thorac Dis. 2020;12(Suppl 1):S16-S21. doi:10.21037/jtd.2019.12.63 
5. Vijayan AL, Vanimaya, Ravindran S, et al. Procalcitonin: a promising diagnostic marker for sepsis and antibiotic therapy. $J$ Intensive Care. 2017;5:51. Published 2017 Aug 3. doi:10.1186/s40560-017-0246-8

6. Morgan J, Meltzer $M$ et al: excess mortality, hospital stay, and cost due to candidemia: a case-control study using data from population-based candidemia surveillance, Infect Control Hosp Epidemiol 2005 jun: 540-547. doi: 10.1086/502581.

7. Blot SI, Vandewoude KH, Hoste EA, et al. Effects of nosocomial candidemia on outcomes of critically ill patients. Am J Med 2002;113(6):480-5.

8. Ostrosky-Zeichner L, Sable C, Sobel J, et al. Multicenter retrospective development and validation of a clinical prediction rule for nosocomial invasive candidiasis in the intensive care setting. Eur J Clin Microbiol Infect Dis 2007;26(4):271-6.

9. Dodds Ashley ES, Lewis R, Lewis JS, Martin C, Andes D: pharmacology of systemic antifungal agents, Clin Infect Dis 2006; 43:S28-39.doi: 10.1086/504492.

10. Pappas PG, Kauffman CA, Andes D, et al. Clinical practice guidelines for the management of candidiasis: 2009 update by the Infectious Diseases Society of America. Clin Infect Dis 2009;48(5):50335. 